\title{
Three-dimensional Laser Scanning Confocal Analysis of Conjunctival Microcysts in Glaucomatous Patients Before and After Trabeculectomy
}

\author{
SILVIO DI STASO ${ }^{1 *}$, LUCA AGNIFILI $^{2 *}$, ANGELA DI GREGORIO ${ }^{1}$, HILARY CLIMASTONE $^{1}$, \\ EMILIO GALASSI ${ }^{1}$, VINCENZO FASANELLA ${ }^{2}$ and MARCO CIANCAGLINI ${ }^{1}$ \\ ${ }^{1}$ Ophthalmology Unit, Department of Life, Health and Environmental Sciences, \\ University of L'Aquila, L'Aquila, Italy; \\ ${ }^{2}$ Ophthalmology Clinic, Department of Medicine and Aging Sciences, \\ University G. d'Annunzio of Chieti-Pescara, Chieti, Italy
}

\begin{abstract}
Background/Aim: In glaucoma, conjunctival epithelial microcysts (CEM) have been extensively investigated by means of laser scanning confocal microscopy. In the present case series, we examined eight glaucomatous patients undergoing trabeculectomy to obtain a 3-dimensional (3-D) characterization of CEM. Materials and Methods: Image acquisition was performed in z-scan automatic volume mode by Heidelberg Retina Tomograph III/Rostock Cornea Module and a series of 40 images of $300 \times 300 \mu \mathrm{m}(384 \times 384$ pixels $)$ to a maximum depth of 40 $\mu m$ were acquired throughout the upper bulbar conjunctiva before (at the site planned for surgery) and eight weeks after trabeculectomy. The 3-D volume tissue reconstruction with maximal size of $300 \times 300 \times 40 \mu \mathrm{m}$ was obtained. Results: In the enface view, CEM appeared as empty, optically clear, round or oval shaped sub-epithelial structures. The 3-D spatial reconstruction showed microcysts as oval-shaped and optically clear elements, which were close, but clearly separated from the epithelium. CEM were embedded in the extra-cellular spaces and located about $10 \mu \mathrm{m}$ below the epithelial surface. After trabeculectomy, CEM increased density and area especially along the horizontal axis. Conclusion: The 3-D in vivo confocal reconstruction of CEM
\end{abstract}

This article is freely accessible online.

*These Authors contributed equally to this work.

Correspondence to: Marco Ciancaglini, Ophthalmology Clinic, Via Lorenzo Natali 1,67100 Coppito, L'Aquila, Italy. Tel: +39 3499778688, e-mail: marco.ciancaglini@cc.univaq.it

Key Words: Confocal microscopy, open angle glaucoma, transscleral aqueous humor outflow, trabeculectomy, conjunctival microcysts, three-dimensional analysis. permits for better clarification of their microscopic anatomy and patho-physiological significance, confirming their involvement in AH flow through the bleb-wall after filtration surgery for glaucoma.

Glaucoma is the second leading cause of blindness worldwide and it is the most frequent cause of irreversible blindness (1). There are two types of adult-onset glaucoma: primary open-angle glaucoma (POAG), in which the angle of the anterior chamber is anatomically open, and primary angle-closure glaucoma (PACG), in which the angle is anatomically closed. POAG is an optic chronic neuropathy caused generally by a rise of intraocular pressure which leads to a progressive retinal ganglion cell apoptosis and visual field loss (2). The goal of POAG treatment is to maintain the patient's visual function with an intra-ocular pressure (IOP) lowering by medical therapy. In patients with advanced glaucoma an aggressive IOP lowering should be obtained using surgical procedure. The most widely performed surgical procedure in POAG is trabeculectomy that produces a protected fistula between the anterior chamber and the subconjunctival space.

The in vivo confocal microscopy (IVCM) is the emerging technique to study the ocular surface structures in patients with glaucoma (3) and laser scanning confocal microscopy (LSCM), which couples the Rostock Cornea Module (RCM) with the Heidelberg Retina Tomograph (HRT II and III; Heidelberg Engineering GmbH, Heidelberg, Germany), represents a major advance compared to the other confocal microscopes since it is capable of imaging either transparent or translucent and semi-opaque structures such as the conjunctiva. For these reasons LSCM is currently the most frequently used confocal microscope in ophthalmology for both research purposes and in daily clinical practice. The interest to study the modification of conjunctival surface in 
glaucomatous eye was focused on the presence of epithelial microcysts (CEM) that is typical but not limited to eyes that underwent filtration surgery, since they were described also in untreated ocular hypertension $(\mathrm{OH})$, in medically-treated open glaucoma and also in bleb-less surgical procedures (4-10).

The in vivo detection of microcysts in such a wide spectrum of glaucoma-related conditions, allowed considering them as an in vivo hallmark of aqueous humor $(\mathrm{AH})$ passage through the sclera and the conjunctiva.

Despite extensively investigated, microcysts were observed in a two-dimensional manner, which is the standard analysis provided by the laser scanning confocal microscopy; nevertheless, this approach presents the limitation of an incomplete architectural definition of microcysts. In the present study, we analyzed the three-dimensional (3-D) features of CEM in patients with open angle glaucoma before and after trabeculectomy, in order to better clarify the morphological characteristics and the patho-physiological significance of these structures.

\section{Materials and Methods}

Patients. This was a case series study that adhered to the tenets of the Declaration of Helsinki. Informed consent was obtained from all subjects after explanation of the methods used in the study and the possible consequences.

A 3-D in vivo microscopic technique using the Heidelberg Retina Tomograph III (HRT III, Heidelberg Engineering, Germany) in combination with an anterior segment adapter (Rostock Cornea Module) was utilized to shift the focal plane into conjunctiva. Technical characteristics of this instrument and the details of conjunctival examination were previously described $(4,11,12)$.

We consecutively examined 8 eyes ( 5 right and 3 left eyes) of 8 Caucasian patients (5 males and 3 females, mean age of $61.0 \pm 3.8$ years, range 50 to 70 years) affected with primary open angle glaucoma (POAG), and scheduled to undergo mitomycin C (MMC) augmented trabeculectomy, as previously described (12).

Patient candidates for surgery underwent a complete baseline ophthalmological assessment including BCVA (ETDRS chart), IOP measurement (Goldmann applanation tonometry), slit lamp anterior segment and dilated fundus examination, visual field test, and IVCM (24-36 h later).

Inclusion criteria were the following: best corrected visual acuity (BCVA) $\geq 8 / 10$, preoperative uncontrolled IOP ( $\geq 22 \mathrm{mmHg}$, mean of three measurements at $9 \mathrm{AM}, 12$ noon and $4 \mathrm{PM}$ ) under maximal tolerated medical therapy (including oral acetazolamide), topical hypotensive therapy started at least 12 months before surgery and unmodified during the last two months, progression of glaucomatous damage confirmed on three consecutive SITA standard 30-2 visual fields (Humphrey field analyzer II 750; Carl Zeiss Meditec, Inc., Dublin, CA, USA). Exclusion criteria were: best corrected visual acuity $\leq 20 / 40$, refractive error $\geq 4$ diopters (spherical equivalent), central corneal thickness $<520$ and $>570 \mu \mathrm{m}$, pseudo-exfoliation, endstage glaucoma (mean deviation $>15 \mathrm{~dB}$ ), visual-field defects attributable to non-glaucomatous conditions, history of angle-closure or an occludable angle on gonioscopy, argon laser trabeculoplasty or laser iridotomy, previous surgical procedure for glaucoma or any history of ocular surgery except cataract, aphakia, signs of inflammatory eye disease, uveitic glaucoma, ocular trauma, dry eye or other ocular disease during the previous 12 months, contact lens wear, any topical therapy except anti-glaucoma eye drops.

Post-surgical therapy required unpreserved topical steroids for 4 weeks (unpreserved dexamethasone $0.15 \%$ eye drops four times a day for 2 weeks and three-time daily for the following 2 weeks) and topical antibiotic for 2 weeks (Ofloxacin $0.3 \%$ fourfold daily). Surgery was considered successful if a third reduction of preoperative IOP without medical treatment, with IOP lower than $18 \mathrm{mmHg}$, was achieved (12).

Patients were scheduled to receive a weekly follow-up in the first month, and monthly in the following months. Baseline and eight weeks data were considered for the statistical analysis.

Confocal microscopy procedure. IVCM examination was performed to analyze the upper bulbar conjunctival epithelium at the site planned for surgery, before and 8 weeks after trabeculectomy (that is 4 weeks after cessation of topical post-operative therapy). The confocal sessions aimed at identifying the presence, density and area of CEM.

At baseline (48-72 h before surgery) a $5 \times 5 \mathrm{~mm}$ area of the upper bulbar conjunctiva, centered at 12 o'clock meridian (2-4 $\mathrm{mm}$ from the limbus) and corresponding to the bleb site after surgery, was analyzed. To explore this site, patients were instructed to direct the gaze downward for the entire duration of the confocal session. Images were acquired within the epithelium (10-40 $\mu \mathrm{m}$ of depth).

Sequential images $300 \times 300 \mu \mathrm{m}$ in size $(384 \times 384$ pixels), derived from automatic scans were acquired of the upper bulbar conjunctiva. An internal scanning device moving the focal plane perpendicularly to the $x-y-p l a n e$, in a similar way to HRT III optic disc topography, was used to obtain the $3-\mathrm{D}$ images. During the image capture process, the $\mathrm{z}$-movement is stopped and the image plane is exactly perpendicular to the $\mathrm{z}$-axis. Image acquisition was performed in $\mathrm{z}$ scan automatic volume mode and a series of maximal 40 images to a maximum depth of $40 \mu \mathrm{m}$ were captured (13). The image acquisition time for a single frame was 0.025 seconds, with a total acquisition time for a 3-D image stack of 1.2 seconds. The automatic brightness mode was selected during examination

To overcome the misalignment of each single frame, a manual image alignment was performed and after this procedure, a 3-D reconstruction of a tissue volume with a maximal size of $300 \times 300 \times 40 \mu \mathrm{m}$ and a voxel size of $0.78 \times 0.78 \times 0.95 \mu \mathrm{m}$ was obtained by using the AMIRA volume-rendering software package (AMIRA 5.4, TGS Inc., USA). Volume orientation for viewing planes and 3-D perspectives segmentation, enface view, determination of distances, cross-sections and surface analysis of CEM were generated to assess the conjunctival epithelium microcysts in sufficiently high quality. After eight weeks, confocal microscopy was repeated to evaluate CEM within the bleb-wall epithelium, taking particular attention to avoid bleb traumatisms. All confocal sessions were performed under topical anesthesia ( $0.4 \%$ oxybuprocaine), by a single experienced operator (VF); confocal examination lasted less than 5 min and no complications related to the procedure were reported.

The 3-D reconstruction of the CEM morphology was the main objective of the study; baseline and eight weeks mean microcyst density and area (MMD and MMA), mean IOP, and mean number of medications were the adjunctive outcome measures. 

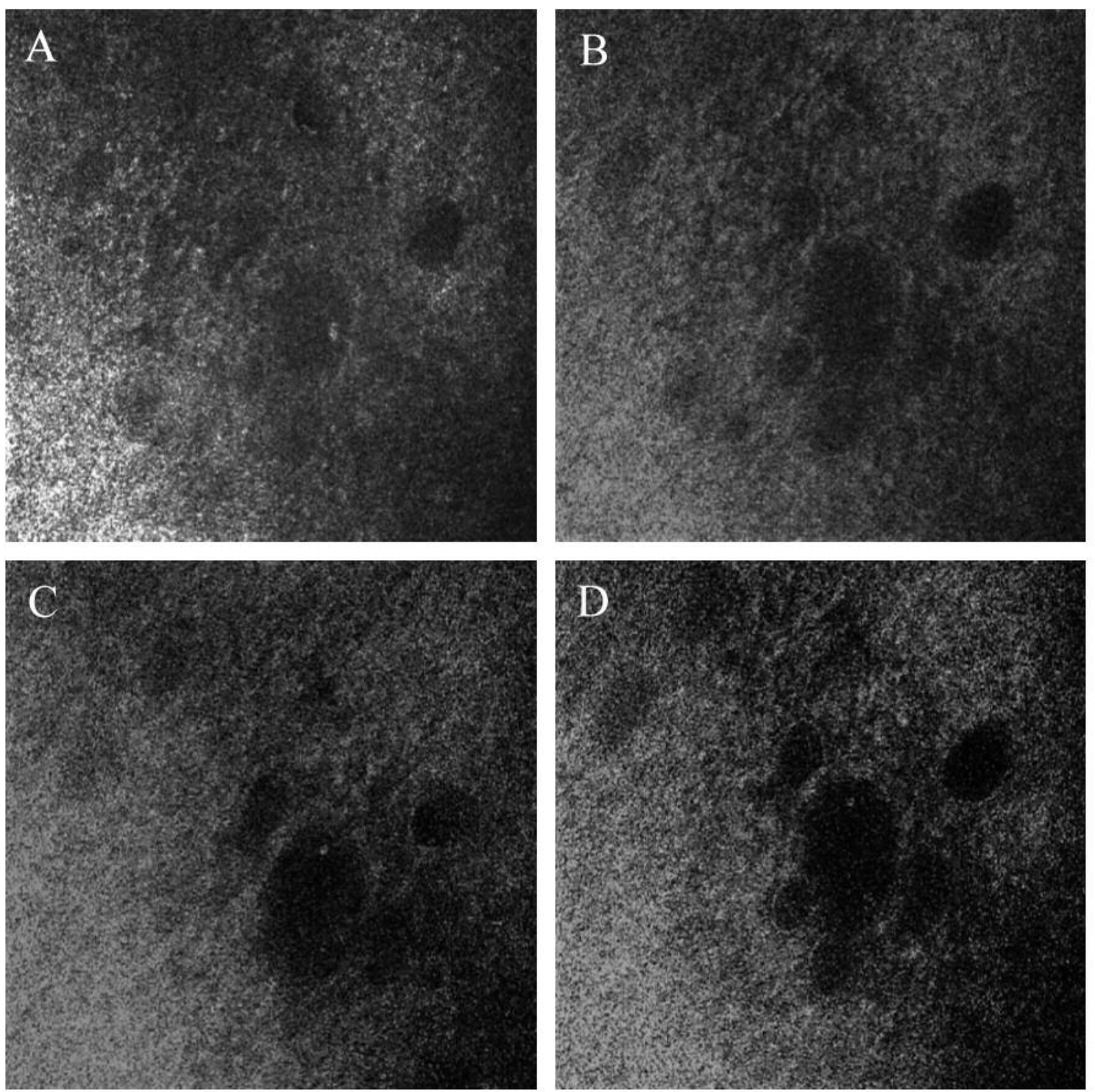

Figure 1. Enface views of upper bulbar conjunctiva with embedded microcysts before surgery, at the surface (A), $10 \mu \mathrm{m}(B), 15 \mu \mathrm{m}(C)$ and $20 \mu \mathrm{m}$ of depth $(D)$.

Statistical analysis. Analysis was performed by SPSS $\AA^{\text {Advanced }}$ Statistical TM 13.0 Software and SPSS Sample Power Software (2005, Chicago, IL, USA). Wilcoxon signed-rank test for paired data was used to assess differences between baseline and eight weeks MMD and MMA; T-test for paired data was used to calculate differences between and eight weeks IOP and number of medications. A $p$-value $<0.05$ was considered statistically significant.

\section{Results}

The morphology of the conjunctival epithelium, along with the characteristics of microcysts and sub-epithelial tissue were clearly depicted by IVCM in all cases.
Filtration surgery was completely successful in all patients at the last follow-up ( 8 weeks): mean baseline IOP reduced from $28.1 \pm 3.12$ to $15.81 \pm 2.4 \mathrm{mmHg}(p<0.01)$, and mean number of medications from $2.9 \pm 0.76$ to $0(p<0.001)$. MMD $\left(\right.$ cysts $\left./ \mathrm{mm}^{2}\right)$ and MMA $\left(\mu \mathrm{m}^{2}\right)$ were $12.71 \pm 3.80$ and 2,516 \pm 388 , respectively, before surgery; eight weeks after surgery MMD and MMA presented a 7- to 10-fold increase, with values of $86.1 \pm 21.3$ and $23,477 \pm 11,874.8$, respectively $(p<0.001)$.

At baseline, in the enface view (Figure 1), CEM appeared as dark structures, round or oval shaped, located below the epithelium and similar to those previously described in medically-treated glaucomatous patients $(4,7,8)$. 

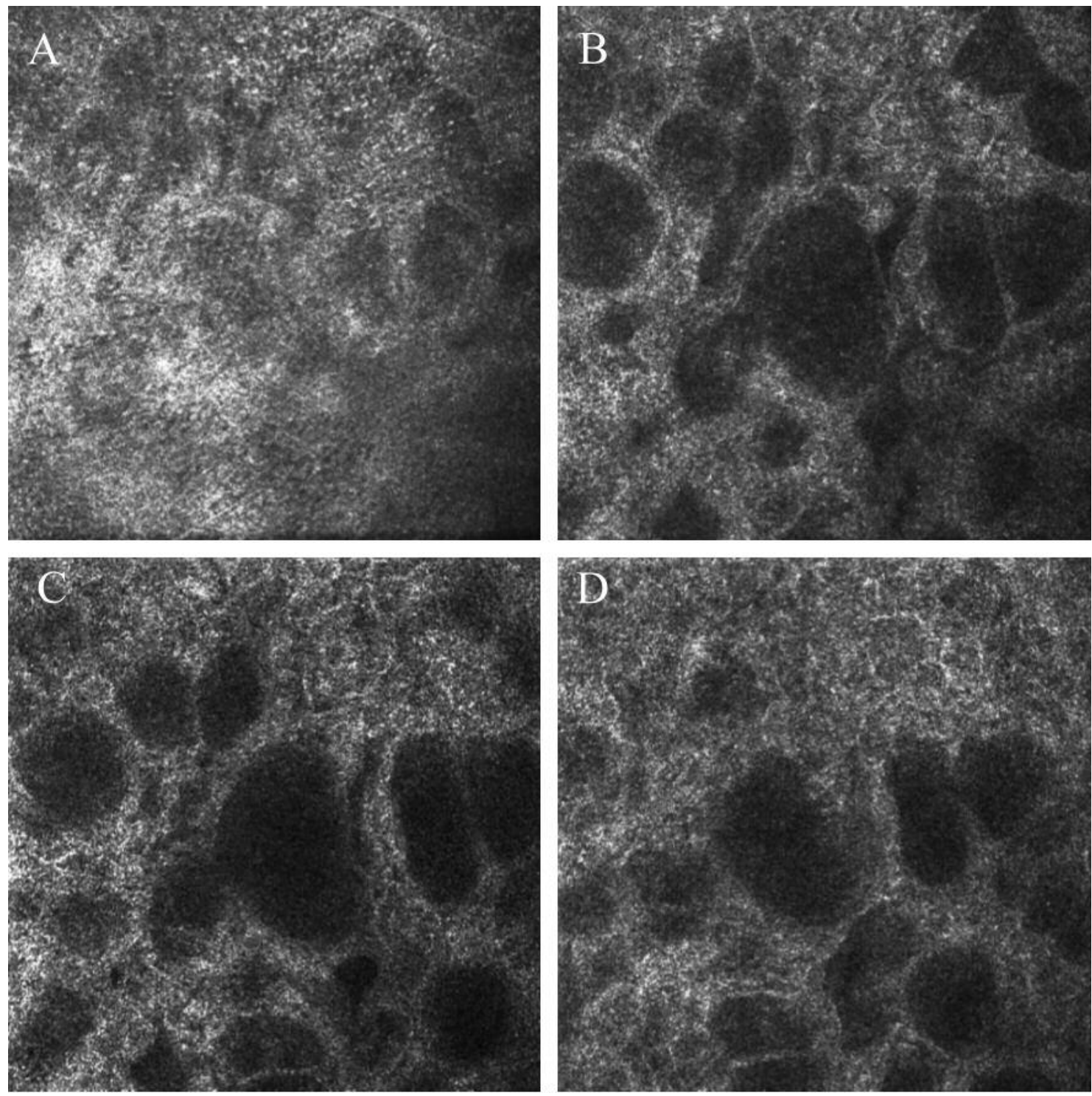

Figure 2. Enface views of upper bulbar conjunctiva eight weeks after trabeculectomy at the surface (A), $10 \mu m(B), 15 \mu m(C)$, and $20 \mu m(D)$, showing a greater number of microcysts, with an increased area at the different focal plans.

Eight weeks after surgery, at the enface view bleb-wall microcysts (Figure 2) presented features mildly different compared to baseline, showing larger size, occasionally filled with debris or presumably inflammatory cells, usually without capsule, sometimes confluent or clustered.

At the 3-D analysis, all microcysts before and after surgery appeared located in the extra cellular matrix, about $10 \mu \mathrm{m}$ below the epithelial external surface, as previously described (13). The vertical diameter of CEM did not change after surgery, ranging from 20 to $25 \mu \mathrm{m}$ at both baseline and eight weeks (Figure 3). Baseline and eight weeks CEM horizontal diameters differed between them, being larger after surgery
(Figures 4 and 5). These 3-D features explain a preferred horizontal diffusion of microcysts and surface analysis revealed the presence of a single layer of CEM (Figures 5, 6).

In addition, the 3-D analysis clarified the anatomical relationship between microcysts, epithelium and subepithelium: the $\mathrm{z}$ scans revealed that CEM were very close, but clearly separated from the epithelium (Figure 3). The 3$\mathrm{D}$ reconstruction supported the hypothesis that microcysts are volumetric structures, expanding in the sub-epithelial tissue and reaching the deep portion of the epithelium. No extracellular or canal structures were recognized below the microcysts. 

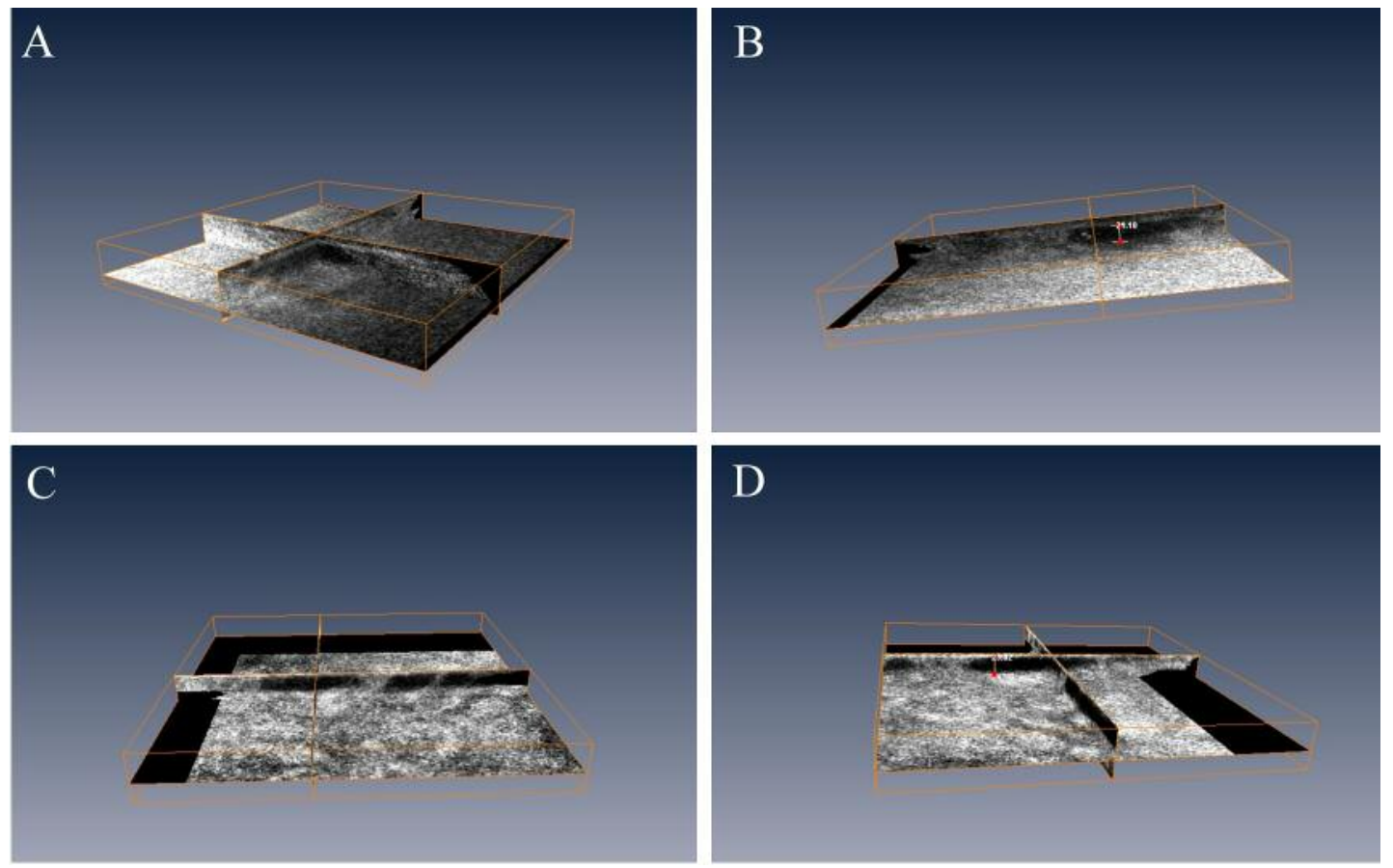

Figure 3. Multiple z scans before $(A, B)$ and after trabeculectomy $(C, D)$ depicts that conjunctival epithelium and microcysts are independent and clearly separated structures with similar eight.

\section{Discussion}

The long-term success of glaucoma filtering surgery is mainly dependent on the development of a functioning filtering bleb. The formation and the maintenance of this functioning bleb, with regard to wound healing and conjunctival scarring, are therefore of primary importance. Clinical examination provides limited information on the drainage ability of a bleb filtering, although the absence of vascularization and tortuous vessels were significantly correlated with good bleb function (14). IVCM is a powerful diagnostic tool, which offers a bridge between clinical and laboratory observations of ocular surface, both in health and disease. At presence, the most widely used IVCM devices are the white light slit scanning confocal microscopy (Confoscan, Nidek Co. Ltd., Gamagori, Japan), which provides lower transverse and axial resolution and a laser scanning confocal microscopy (HRT-III) which uses a coherent high-intensity light source. Because of the highdepth resolution, optical sections of only a few micrometers can be imaged and precisely measured in combination with a high contrast (3). IVCM permitted a major advancement in the AH filtration assessment and supported biomicroscopic evaluation as the mainstay for bleb analysis. The microscopic analysis at a cellular level indicated the presence of microcysts as objective features associated with success or failure $(4,11,12,14-16)$.

During the last decade, this technique documented the presence of CEM also within the conjunctival epithelium of untreated ocular hypertension or medically controlled glaucomatous patients, even if with slightly different features $(4,6,9,12,17)$. The presence of microcysts in eyes that underwent surgery is consistent with an adaptive mechanism, aimed at increasing the $\mathrm{AH}$ movement through the sclera and, finally, conjunctiva. The pathway of $\mathrm{AH}$ in conjunctiva after glaucoma surgery is thought to be either a transvenous route or through the epithelium into tears $(18,19)$. Movement of the AH through the epithelium has been suggested by chemical analysis of bleb fluid, demonstrating ascorbic acid levels similar to those of aqueous humor. The 3-D homogeneous hypo-reflectivity of the whole microcyst, seems to further support the hypothesis that each microcyst is filled with fluid.

Amar et al. (20) analysed the transconjunctival pathway of the AH through the bleb using IVCM and immunohistology on impression cytology, and demonstrated conjunctival goblet cell (GCs) changes, suggesting that these cells correspond to the microcysts observed in functioning blebs with in vivo confocal microscopy. In our study, the 3-D analysis confirms that the features of CEM are very similar to those of GCs. While goblet 


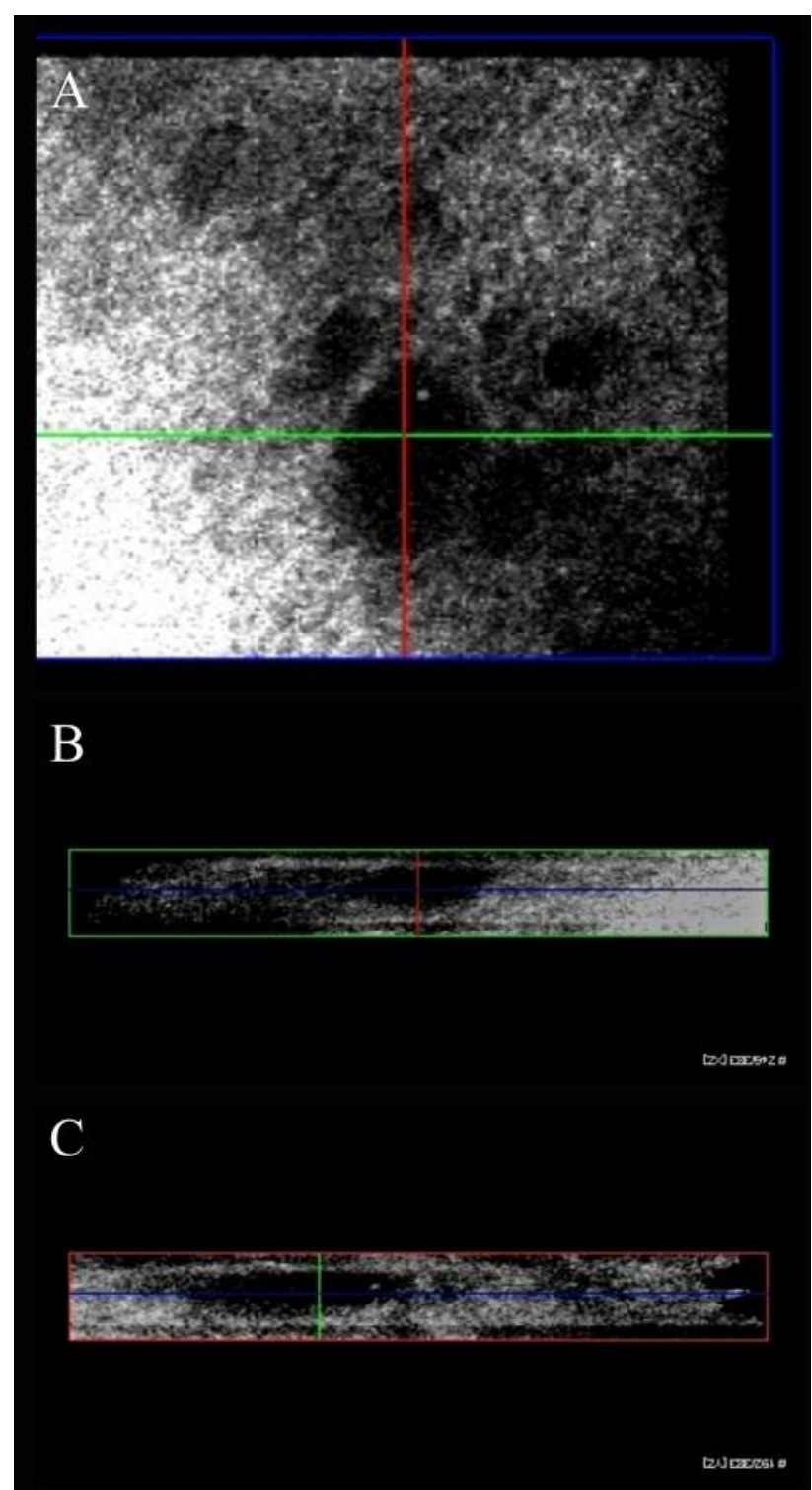

Figure 4. 3-D analysis at the intermediate layer of conjunctiva before surgery $(A)$, showing small oval shaped microcysts in $Y(B$, red line $)$ and $X$ scans $(C$, green line). The blue line identifies the scan plane with respect to the surface (top image).

cells in human conjunctiva are intercalated between the epithelial cells with regional variation in their density and organized in a single layer, on the other hand microcysts are located very close under the epithelium and always arranged in one layer. Furthermore, Doughty (21) visualized GCs in impression cytology specimens from bulbar conjunctiva of the rabbit eye using Giemsa staining and found that goblet cells were ellipsoid in shape, the longest dimension averaged 16.7 $\pm 2.3 \mu \mathrm{m}$, the shortest dimension averaged $14.4 \pm 1.8 \mu \mathrm{m}$

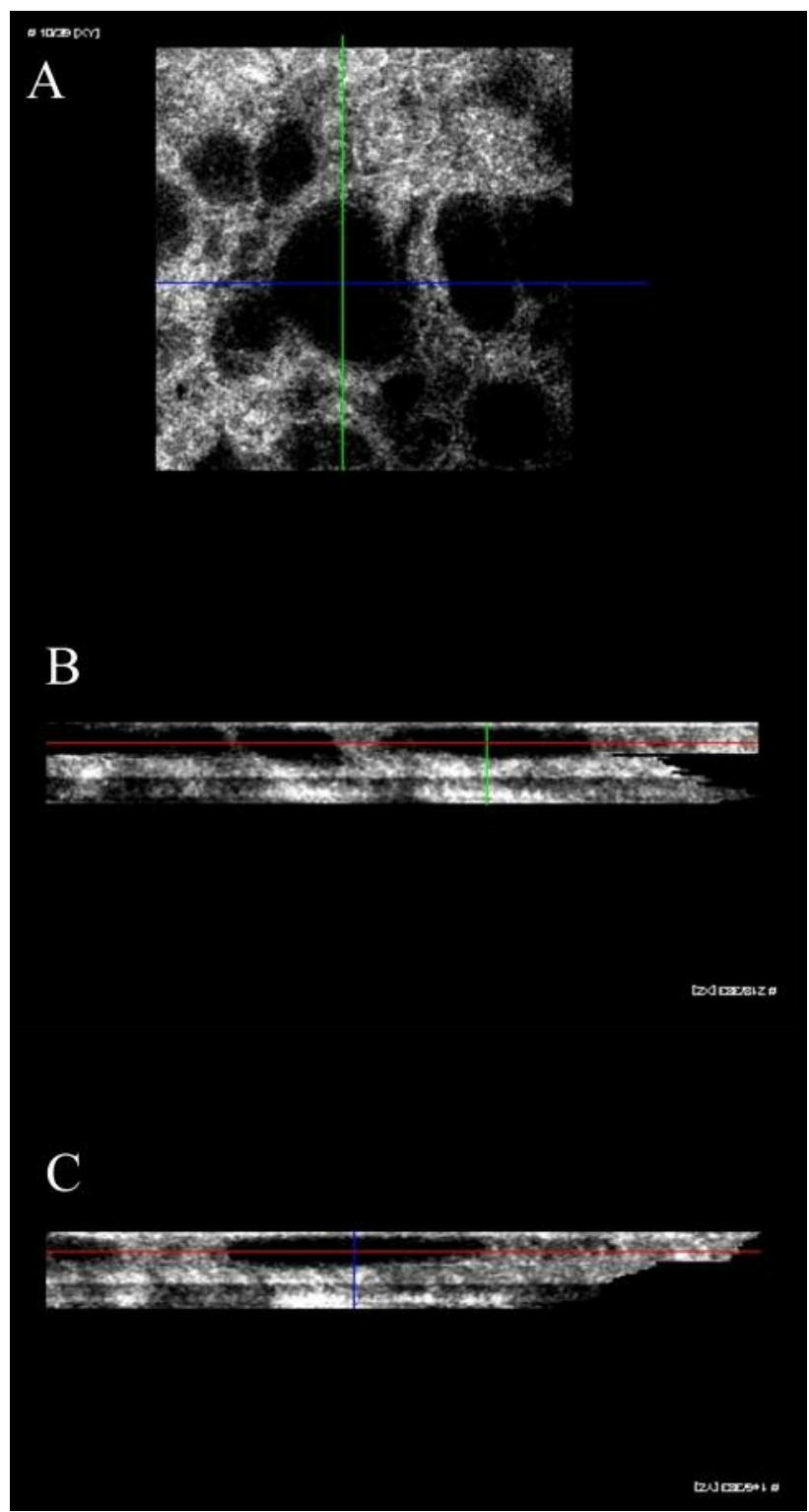

Figure 5. 3-D analysis at the intermediate layer of conjunctiva after surgery $(A)$, showing oval shaped microcysts in $Y(B$, red line) and $X$ scans $(C$, green line) with greater density and larger area compared to the pre-surgical examination. The blue line identifies the scan plane with respect to the surface (top image).

and GCs area averaged $193 \pm 50 \mu \mathrm{m}^{2}$. Z scans and surface analysis of IVCM indicate that CEM had an ellipsoid shape with similar eight before and after surgery and larger horizontal diameter, especially in eyes after trabeculectomy, where a great increase of microcysts area was found. In addition, the increased horizontal diameter of CEM in combination with the similar vertical diameter after surgery, lead to suppose that $\mathrm{AH}$ tends to diffuse easier horizontally because of different inner hydraulic resistivity. Recently, (22) the preoperative 

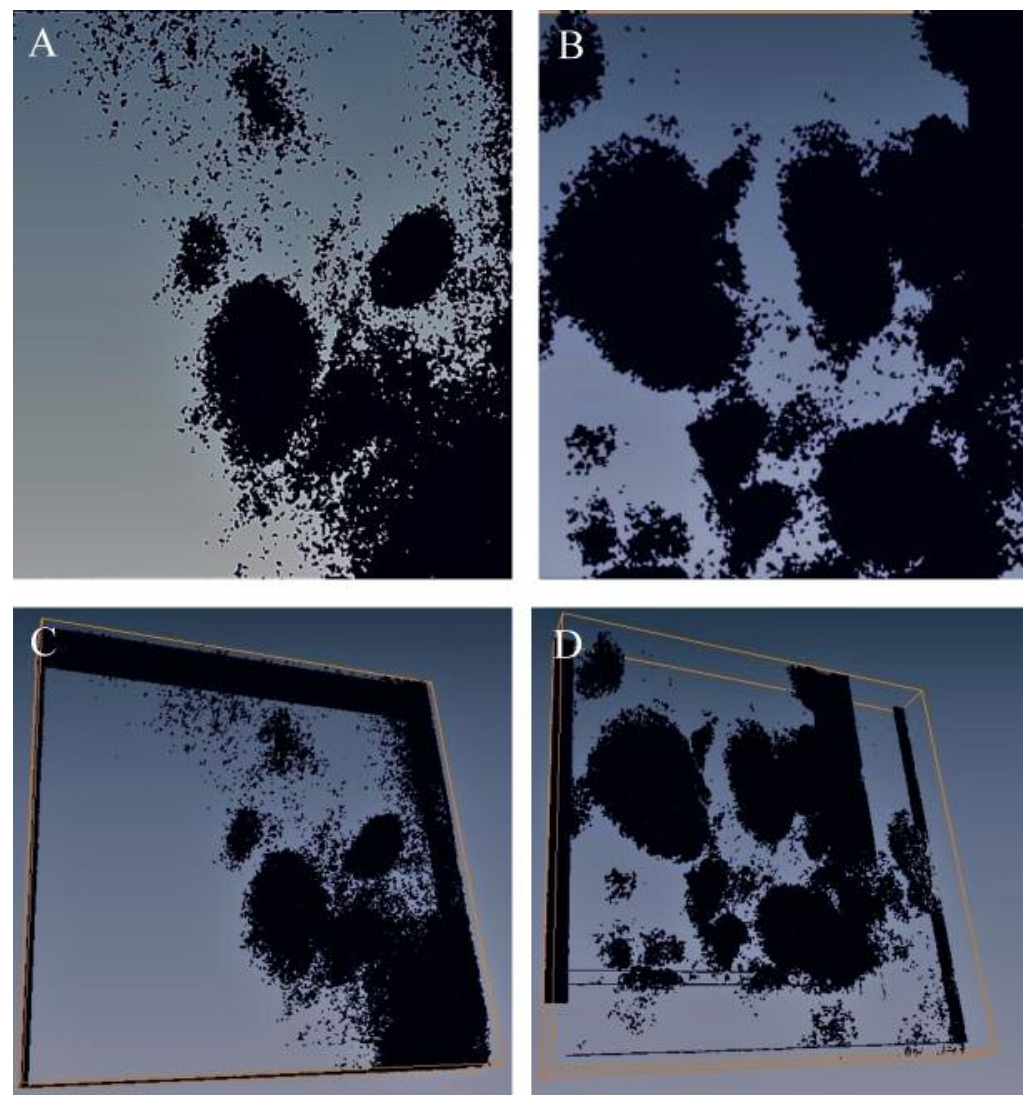

Figure 6. Enface and oblique view of surface analysis of microcysts before $(A, C)$ and after trabeculectomy $(B, D)$ confirm the presence of a monolayer of CEM.

conjunctival GCs density together with dendritic cell density and stromal meshwork reflectivity were correlated with the filtration surgery outcome. Independently from the origin, CEM be intended as active structures, with the intent to transport the AH towards the ocular surface. The different 2-D and 3-D morphological aspects of CEM in glaucomatous eyes before and after trabeculectomy, could derive from the $\mathrm{AH}$ passage through thinner sclera, bleb cavity and bleb-wall tissues and may account for the greater dimension and for the not infrequent clustering tendency of CEM.

The present study presents some limitations, mostly related to the complexity of the $3-\mathrm{D}$ analysis process. The image capture and data examination require a well-trained operator to obtain high quality images because the 3-D analysis is complex and a time-consuming procedure. Therefore, only a small group of patients was enrolled in the study. Another problem is the misalignment of images occurring during capture due to eye movement that may be corrected with manual intervention; in the worst case, when the alignment process fails, part of the individual slices leaves the region of interest and a dark area appears (Figure 3C and D). At the light of these limitations the 3-D reconstruction of ocular surface should be considered an experimental procedure not applicable to date in the clinical practice.

In conclusion, this technique significantly improves the information of the 2-D imaging, provides further data about transcleral flow of $\mathrm{AH}$ and highlights the crucial role of microcysts as a predictive marker of functioning blebs.

\section{References}

1 Quigley HA and Broman AT: The number of people with glaucoma worldwide in 2010 and 2020. Br J Ophthalmol 90: 262-267, 2006

2 Quigley HA: Glaucoma. Lancet 377: 1367-1377, 2011.

3 Villani E, Baudouin C, Efron N, Hamrah P, Kojima T, Patel SV, Pflugfelder SC, Zhivov A, and Dogru M. In vivo confocal microscopy of the ocular surface: from bench to bedside. Curr Eye Res 39: 213-231, 2014.

4 Ciancaglini M, Carpineto P, Agnifili L, Nubile M, Lanzini M, Fasanella V and Mastropasqua L: Filtering bleb functionality: a clinical, anterior segment optical coherence tomography and in vivo confocal microscopy study. J Glaucoma 17: 308-317, 2008. 
5 Mastropasqua L, Agnifili L, Ciancaglini M, Nubile M, Carpineto P, Fasanella V, Figus M, Lazzeri S and Nardi M: In vivo analysis of conjunctiva in gold micro shunt implantation for glaucoma. Br J Ophthalmol 94: 1592-1596, 2010.

6 Agnifili L, Carpineto P, Fasanella V, Mastropasqua R, Zappacosta A, Di Staso S, Costagliola C and Mastropasqua L: Conjunctival findings in hyperbaric and low-tension glaucoma: an in vivo confocal microscopy study. Acta Ophthalmol 90: 132-137, 2012.

7 Mastropasqua L, Agnifili L, Salvetat ML, Ciancaglini M, Fasanella V, Nubile M, Mastropasqua R, Zeppieri R and Brusini $\mathrm{P}$ : In vivo analysis of conjunctiva in canaloplasty for glaucoma. Br J Ophthalmol 96: 634-639, 2012.

8 Mastropasqua L, Agnifili L, Mastropasqua R, Fasanella V, Nubile M, Toto L, Carpineto $\mathrm{P}$ and Ciancaglini $\mathrm{M}$ : In vivo laser scanning confocal microscopy of the ocular surface in glaucoma. Microsc Microanal 20: 879-894, 2014.

9 Mastropasqua R, Fasanella V, Pedrotti E, Lanzini M, Di Staso S, Mastropasqua L and Agnifili L: Trans-conjunctival aqueous humor outflow in glaucomatous patients treated with prostaglandin analogues: an in vivo confocal microscopy study. Graefes Arch Clin Exp Ophthalmol 252: 1469-1476, 2014.

10 Mastropasqua R, Agnifili L, Fasanella V, Toto L, Brescia L, Di Staso S, Doronzo E and Marchini G: Uveo-scleral outflow pathways after ultrasonic cyclocoagulation in refractory glaucoma: an anterior segment optical coherence tomography and in vivo confocal study. Br J Ophthalmol 100: 1668-1675, 2016.

11 Guthoff R, Klink T, Schlunck G and Grehn F: In vivo confocal microscopy of failing and functioning filtering blebs: results and clinical correlations. J Glaucoma 15: 552-558, 2006.

12 Ciancaglini M, Carpineto P, Agnifili L, Nubile M, Fasanella V, Mattei PA and Mastropasqua L: Conjunctival characteristics in primary open-angle glaucoma and modifications induced by trabeculectomy with mitomycin $\mathrm{C}$ : an in vivo confocal microscopy study. Br J Ophthalmol 93: 1204-1209, 2009.

13 Di Staso S, Ciancaglini M, Agnifili L, Fasanella V, Nubile M, Mastropasqua R, Galassi $\mathrm{E}$ and Mastropasqua L: In vivo threedimensional analysis of conjunctival epithelial microcysts in glaucoma. J Clin Exp Ophthalmol 7: 1-7, 2016.

14 Caglar C, Karpuzoglu N, Batur M and Yasar T: In vivo confocal microscopy and biomicroscopy of filtering blebs after trabeculectomy. J Glaucoma 25: 377-383, 2016.
15 Labbé A, Dupas B, Hamard P and Baudouin C: In vivo confocal microscopy study of blebs after filtering surgery. Ophthalmology 112: 1979-1986, 2005.

16 Messmer EM, Zapp DM, Mackert MJ, Thiel M and Kampik A: In vivo confocal microscopy of filtering blebs after trabeculectomy. Arch Ophthalmo 124: 1095-1103, 2006.

17 Mastropasqua L, Agnifili L, Mastropasqua R and Fasanella V: Conjunctival modifications induced by medical and surgical therapies in patients with glaucoma. Curr Opin Pharmacol 13: 5664, 2013.

18 Tenner A and Jaeger W: Demonstration of the drainage of aqueoushumor following various glaucoma operations by means of injection of fluorescein into the anterior chamber. Ber Zusammenkunft Dtsch Ophthalmol Ges 74: 853-855, 1977.

19 Kronfeld PC: The chemical demonstration of transconjunctival passage of aqueous after antiglaucomatous operations. Am J Ophthalmol 35: 38-45, 1952.

20 Amar N, Labbè A, Hamard P, Dupas B and Baudouin C: Filtering blebs and aqueous pathway. An immunocytological and in vivo confocal microscopy study. Ophthalmology 115: 11541161,2008

21 Doughty MJ: Giemsa-based cytological assessment of area, shape and nucleus:cytoplasm ratio of goblet cells of rabbit bulbar conjunctiva. Biotech Histochem 91: 501-509, 2016.

22 Mastropasqua R, Fasanella V, Brescia L, Oddone F, Mariotti C, Di Staso $\mathrm{S}$ and Agnifili L: In vivo confocal imaging of the conjunctiva as a predictive tool for the glaucoma filtration surgery outcome. Invest Ophthalmol Vis Sci 58: 114-120, 2017.
Received August 17, 2017

Revised September 14, 2017

Accepted September 19, 2017 